\title{
DIDACTOEUGENIA. LA BUENA PRÁCTICA DOCENTE EN LA ENSEÑANZA
}

\section{DIDACTOEUGENIA. TEACHER'S GOOD PRACTICE IN TEACHING}

Margarita Espinosa Jiménez

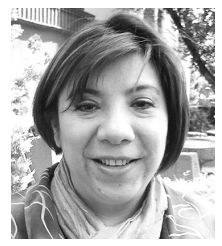

Profesora en Educación Primaria, Normal La Florida, México. Licenciada en la Atención de Personas con Trastornos de Audición y Lenguaje, Instituto Superior de Docentes en Educación Especial, México. Maestra en Enseñanza Superior, Universidad La Salle, México. Doctorado en Pedagogía, UPAEP. Se ha desempeñado en la docencia en todos los niveles educativos; ha formado parte de consejos consultivos como asesoría educativa en el DF y en diferentes Estados de la República. Desde 1991 es docente en diferentes universidades en nivel superior. Profesora invitada en clases de posgrado en Universidades del DF e interior de la República. Perteneciente al Comité de Reactivos del EGEL Pedagogía y ciencias de la Educación de Ceneval. Correo electrónico: [mejimene@up.edu.mx].

\section{RESUMEN}

La función emancipadora de la educación desarrolla el gusto y el entusiasmo por aprender más allá de lo inmediato (textos, contextos y acciones docentes). La actividad del profesor busca la apropiación del conocimiento; pero también por ese actuar, se pone en práctica lo que ese profesional es y que influye en el estudiante circunscrito en el ámbito escolar. 
Los docentes que a través de su enseñanza, se preocupan por sus estudiantes, son cercanos a ellos, los apoyan para conformar significados, se entusiasman al impartir sus clases e influyen en el aprender y estudiar para conocer, son quienes enseñan el valor de la reflexión, de conocer más allá de una calificación, sólo por el gusto de saber, muestran un mundo nuevo, dan cauce y conforman estructuras de conocimiento que permiten mejorar a sus estudiantes. Ellos representan lo que puede concebirse como un buena práctica docente que promueve el aprendizaje y que no genera la obstrucción del mismo. La intención de esta nota es argumentar el valor educativo de la enseñanza, promoviendo a las didactoeugenias.

Palabras clave: Docente; valor educativo de la enseñanza; didactoeugenias.

\section{ABSTRACT}

The emancipatory function, which has the education, requires the development of the taste and enthusiasm for learning beyond the immediate, what is presented in the texts, contexts and educational activities; this teacher's activity that continually seeks to support the acquisition of knowledge; but also by that act, it is put into practice what the professional is that influences student circumscribed in schools. Teaching is the activity and the process generated by the teachers; some are deeply concerned about the content, for generate actions that facilitate the learning of their students; others, for various reasons, have altered the desire to continue learning and knowing. In a special place for learners there are teachers who, through their teaching, did perceive their concern for them, manifested in their area and support when they were aware that there was an obstacle in the creation of meaning; enthusiasm in imparting living in their classes and that instilled in learning and study to know it showed. These are the teachers who teach the value of reflection, to know beyond a rating, just for the pleasure of knowing, are those that show a new world and give up the channel and knowledge structures that enhance their students; are those with what can be conceived as a good teaching practice that promotes learning and does not generate the same blockage. The intention of this paper therefore is to argue the educational value of teaching, promoting the «didactoeugenias».

Key words: Teacher; teaching education value; didactoeugenias. 


\section{INTRODUCCIÓN}

La función emancipadora que posee la educación requiere desarrollar el gusto y el entusiasmo por aprender más allá de lo inmediato, de lo que se presenta en los textos, contextos y las acciones docentes. Esta actividad del profesor busca apoyar continuamente la apropiación del conocimiento; pero también por ese actuar, se pone en práctica lo que ese profesional es y ello influye en el estudiante circunscrito en el ámbito escolar. La enseñanza es la actividad y el proceso que generan los docentes; algunos se encuentran profundamente preocupados por los contenidos, por generar acciones que posibiliten el aprendizaje de sus alumnos; otros, por diversas circunstancias, han alterado el deseo de seguir aprendiendo y conociendo.

En un lugar especial para los educandos se encuentran aquellos docentes que, a través de su enseñanza, les hicieron percibir su preocupación por ellos, manifestada en su cercanía y apoyo cuando se percataban que existía un obstáculo en la conformación de significados; se notaba el entusiasmo que vivían al impartir sus clases y que infundían en el aprender y estudiar para conocer. Éstos son los docentes que enseñan el valor de la reflexión, de conocer más allá de una calificación, sólo por el gusto de saber; son aquellos que muestran un mundo nuevo y que dan cauce y conforman estructuras de conocimiento que permiten mejorar a sus estudiantes; son los que presentan lo que puede concebirse como un buena práctica docente que promueve el aprendizaje y que no genera la obstrucción del mismo.

La intención de esta nota, por ende, es argumentar el valor educativo de la enseñanza, promoviendo a las didactoeugenias.

\section{EL VALOR EDUCATIVO DE LA ENSEÑANZA}

El conocimiento conformado más allá del nivel sensible, requiere salir de la interrelación espontánea y natural de la persona con el objeto de conocimiento y sus vivencias; necesita centrarse en un proceso de planeación. Éste es uno de los momentos característicos que contempla la educación institucional o formal - la escuela- que, como institución educativa, distingue, posibilita y avala los procesos educativos formales con el fin de conformar el conocimiento 
conceptual-actitudinal y procedimental de la persona que comprende diversas finalidades, entre ellas, promover conocimientos no sensibles y holísticos, desde una actividad fundamental: enseñar a partir de una práctica docente ${ }^{1}$ adecuada.

Etimológicamente, enseñar viene del latín in-signare, que significa señal, signo, y que alude a mostrar una señal al otro; facilitar, por medio de un signo, lo que se quiere mostrar. Así, puede afirmarse que la enseñanza, desde un primer acercamiento, implica mostrar o comunicar de la manera más fácil al otro lo que se requiere que conozca con la finalidad de que se lo apropie, retenga y proyecte. Es una acción desarrollada con la intención de llevar al otro a un aprendizaje y, por ende, a un conocimiento; esta actividad y proceso posee una gran influencia en la persona.

Es innegable la trascendencia del proceso educativo escolar con la enseñanza ${ }^{2}$ para el desarrollo de la persona, siendo ésta una de las actividades docentes que realiza el profesor en el contexto de la educación formal, que puede conceptuarse desde diferentes perspectivas (Pla i Molins, M., 1997: 87-92):

Desde el proceso descriptivo de los vocablos, puede indicarse que enseñanza se asocia al contexto de la didáctica, procedente del alemán

1 Rosario Muñoz (2011: 17) comprende la práctica de los profesores como «[...] un proceso de construcción y de redescubrimiento de saberes científicos y profesionales para el desempeño de una práctica profesional: la docencia».

La docencia por ende, es una actividad compleja, puesto que no sólo implica el dominio del contenido a enseñar, sino que requiere además comprender el contexto educativo y psicopedagógico, la materia a impartir, así como las características de los educandos con los que va a interactuar con el fin de apoyar el desarrollo de sus habilidades y procesos.

El objetivo de la docencia por ello es la «[...] autorrealización del estudiante y no la acumulación de contenidos» (Tomás i Folch, 2007: 90).

2 Enseñanza no es lo mismo que instrucción y adoctrinamiento; la instrucción se entiende como la acción docente que se centra en la formación intelectual, así Pacios (1980) la define como la actividad por medio de la cual se adquiere ciencia; el adoctrinamiento consiste en inculcar posturas de forma dogmática y acrítica; cabe recordar que desde el pensamiento ilustrado que da pie a la pedagogía moderna, se busca en la enseñanza la autonomía y la criticidad del estudiante ante los procesos enseñados. 
antiguo teik, y del sánscrito dic con un significado de mostrar; al relacionarse con el significado descriptivo de insignare de la lengua romance y asociado a las voces de la lengua inglesa de teach y learn, así como del alemán lehren, lernen, se avala una enseñanza con intención de promover algo, para que el otro aprenda. Así, la enseñanza implica que alguien debe aprender por lo que se le muestra: mostrar de la mejor forma al otro.

La enseñanza se relaciona con el logro, pues la actividad desempeñada a partir de diversas tareas pretende que el otro alcance lo propuesto. En la acción de enseñanza como actividad se encuentra circunscrito el proceso del resultado.

La enseñanza posee un sentido de intencionalidad que implica un comportamiento por parte del docente para ayudar a la apropiación personal del aprendizaje a partir de sus acciones y tareas; a la vez, esta perspectiva se relaciona con comprender el pensamiento del docente y saber si su actuar se corresponde con su pensar: entender lo que el profesor piensa es equivalente a entender su actuar. Así, cuando el docente organiza su enseñar con la intención de que el otro aprenda, su forma de actuar a partir de esta intención permitirá que la enseñanza se lleve a cabo. No existe enseñanza sin intención educativa.

Toda enseñanza a partir de un carácter normativo, tomará en cuenta criterios de inclusión (en cuanto hay que considerar la igualdad de condiciones para cada estudiante) (Gadoti, 2003), pues de no considerarse, puede llevar a la exclusión dentro de la tarea de enseñar (es relevante considerar factores dialógicos y de integración, así como la noosfera de cada alumno), partiendo de la base de que la enseñanza debe apegarse a un proceso ético.

La enseñanza trasciende; es una actividad que implica tareas entre dos o más personas y exige un sentido de relación, que se equipara a un compromiso y a una responsabilidad del que enseña y del que aprende, dado que en este proceso surge lo que Derek y Mercer (1988), así como Moore (1973), han llamado «conocimiento compartido», en donde se construye dialógicamente el sentido y el significado de contextuar, argumentar, negociar y aplicar lo que se conoció. 
El concepto formal de enseñanza como la actividad que realiza el docente y que se lleva a cabo en la educación formal - estructurada como un conjunto de procesos que implican una tarea de comunicación y reflexión de lo que se muestra, y que permiten al que aprende conformar conocimientos a partir de tareas y acciones de aprendizaje-, no debe de centrarse en la «transmisión» de contenidos o informaciones, sino en el desarrollo de los procesos que potencian la capacidad de aprender. Considerar las operaciones mentales a desarrollar es de vital trascendencia en la enseñanza.

La enseñanza como proceso y como actividad, es tarea del docente que requiere postular entornos que promuevan tareas y acciones de aprendizaje, de manera que al estudiante se le posibilite conformar su propia comprensión de la información, generando significados en situaciones de estudio y colaboración con otros.

La enseñanza, por tanto, abarca acciones más complejas que el sólo estar frente a un grupo intercambiando informaciones; avala una serie de funciones y prácticas dentro del proceso docente que implican estudio, conocimiento e investigación con el fin de que el otro apren$\mathrm{da}$, aunque para ello el docente requiere desarrollar habilidades en su aprender y conocer que pueda perfilar al grupo o los grupos a su cargo.

Es esencial considerar la función de la enseñanza, dado que este proceso — como se analizó- implica y manifiesta las informaciones académicas para que el estudiante conforme significados, siendo su primordial función promover el aprendizaje del otro en un contexto formal. Por ello, enseñar, es un acto didáctico ${ }^{3}$.

La enseñanza debe considerar tres características que darán lugar a sus acciones y tareas:

3 El acto didáctico debe considerarse como la acción intencional que tiene el docente cuando establece una relación comunicativa, que inicia con la enseñanza y termina con el aprendizaje, buscando una significación y significatividad. 
- Sistematización y dirección hacia el logro de propósitos ${ }^{4}$ y competencias ${ }^{5}$. Las tareas y acciones se centrarán en la claridad de lo que se busca alcanzar, formulando adecuadamente los propósitos y las competencias que permitan desarrollar acciones que posibiliten el logro y ubicar los medios adecuados para que éstos se alcancen.

- Estructurar sistemáticamente la enseñanza, considerando en ello la secuencia de contenidos y su desarrollo, métodos y recursos apropiados, ubicar planes de acción que partan de acciones concretas, así como seleccionar las mejores condiciones para desarrollar la tarea de enseñanza.

- Cohesión en los momentos y elementos didácticos ${ }^{6}$.

Con base en Contreras (1990: 23), la enseñanza es: [...] un sistema de comunicación intencional que se [...] genera [...] en un marco institucional y en el que en general se dan estrategias encaminadas a $[\ldots]$ posibilitar [...] el aprendizaje» ${ }^{7}$. Es relevante, ante este concepto, considerar que no sólo puede plantearse como un proceso de comunicación, sino también como un proceso social y situado, que incide en la conformación de las estructuras mentales del estudiante (esquemas y representaciones), a partir de diversas acciones, incididas por los procesos didácticos de la enseñanza.

Con el fin de promover, en el acto de enseñar, el manejo de significados es relevante contemplar, independientemente de las condiciones de enseñanza, las que requiere tener el estudiante en este proceso, de

4 El propósito se entiende como un conocimiento declarativo, procedimental o actitudinal a alcanzar posteriormente. Su redacción implica una acción futura y describe el contexto de lo que requiere lograr; los propósitos exigen diferentes procesos para su logro y se estructuran en función de lo que ha de alcanzar el estudiante.

Los objetivos suelen implicar un resultado que es consecuente con las acciones del proceso de enseñanza y de aprendizaje, identificado generalmente en una conducta que alude a situaciones observables (Morales, 2009).

5 La competencia se entiende como el desempeño que tiene una persona sobre la realidad a partir de su interacción con los otros y el medio, poniendo en función sus conocimientos y procesos cognitivo-práxicos, así como la integración de valores y actitudes; cabe indicar que las competencias son observables a partir de la actuación y el desempeño.

6 Dados a conocer en la teoría de enseñanza propuesta por Herbart.

7 Éste involucra procesos de interacción cognitivo-afectivo y social (Castejón y Navas, 2009). 
tal forma que la comunicación intencional que se involucra se presente de la mejor manera.

Desde la experiencia, las condiciones de enseñanza permiten señalar que el docente necesita tomar en cuenta los factores internos y externos, fundamento en su inicio del proceso de aprender, las condiciones de aprendizaje en que se inserta en la enseñanza, así como el desempeño que posee y pretende alcanzar. Así, al docente le corresponde llevar al ámbito del aula o al contexto áulico la buena práctica de su enseñanza (didactoeugenias).

\section{LA CLASE DEL ACTO DE ENSEÑAR: LAS DIDACTOEUGENIAS}

Este término parte de considerar a la enseñanza como un acto de bondad y de preocupación por el otro; hace referencia al bien que se gesta, en la persona que busca aprender, por las acciones del docente que lo acompaña en este proceso. Viene del latín didakticos, apto para la docencia, eu, bien y genia, generar, animar, motivar. Por ello la didactoeugenia se centra en la práctica buena de enseñanza, dado que busca conseguir un bien por medio de la didáctica, a través de la enseñanza.

La consideración de este término parte del estudio de las investigaciones centradas en el área médica que contempla la iatrogenia, y que da pie a la consideración de conceptos tales como la didactopatía $\left(\right.$ Orantes, 2003) ${ }^{8}$, iatrogenia docente ${ }^{9}$ y la didactogenia ${ }^{10}$.

El estudio de los conceptos analizados, así como la impericia docente y la enseñanza obstructiva, plantean el concepto de dejar de lado, en el acto de enseñar, las didactoeugenias y las didactodamnungenias ${ }^{11}$.

8 El término viene del griego patos, enfermedad y didakticós, enseñanza.

9 Iatrogenia es un término común en la práctica médica que hace referencia al error producido por el médico en su práctica y el daño que puede generar; viene del griego iatros, médico y génesis, crear. La iatrogenia docente abordaría los errores que se presentarían en los estudiantes, derivados de la práctica docente por la misma personalidad de profesor, así como la repetición de prácticas en este acto áulico.

10 Término propuesto por Cukier (2000) que alude al maltrato docente.

11 Este concepto viene del griego damnun, daño, castigo, del latín didaktico, apto para la enseñanza y genia, generar, provocar. Provocar un daño a partir de la enseñanza. 
Frente al grupo, el docente requiere trabajar con su saber reflexivo, dado que en su acción de enseñar debe considerar las características del estudiante, las condiciones de la disciplina o área del conocimiento en el que va a trabajar, así como el marco de la noosfera y del contexto sociocultural; lo que estará interrelacionado con el pensamiento interdisciplinar del profesor (Alsina y Planas, 2008).

En las didactoeugenias, además del saber reflexivo docente, habrá que retomar los indicadores de la actividad de enseñanza buena: contextualizar la enseñanza a partir de las experiencias del estudiante ${ }^{12}$; plantear situaciones que posibiliten el pensamiento complejo ${ }^{13}$ y la inteligencia líquida; generar entornos que permitan el diálogo y la conformación de significados; desarrollar el lenguaje y realizar acciones conjuntas entre docente y estudiantes, así como entre estudiantes y estudiantes (lo que propiciará el aprendizaje de ambos actores); así como el análisis de lo que se enseña y cómo se enseña, y lo que se aprende y cómo se aprende para conformar significados ${ }^{14}$.

Es relevante señalar que las didactoeugenias inducen a un acto de reflexión profunda por parte del docente para realizar su actividad de enseñar, que lo llevarán a buenas prácticas docentes y a generar bienes académicos a sus estudiantes.

\section{CONCLUSIONES}

El generar un bien por medio de la enseñanza exige un docente centrado en su labor, preocupado por conocer lo que enseña y que a partir de ello desarrolla, en los estudiantes, acciones que lleven a interactuar con el objeto de conocimiento para su comprensión; reflexionar en su disciplina; adaptar las acciones de enseñanza, en lo posible, para cada grupo de alumnos; valorar sus avances y las áreas de oportunidad que requieren reforzarse; comunicarse con sus discípulos dentro y fuera del

12 Aludiendo a la inteligencia cristalizada, término generado por Raymond B. Cattrell (Gento, 2010; Rodríguez, 2014).

13 Así como la inteligencia líquida, concepto trabajado por Catrell.

14 Se sugiere para profundizar la temática revisar el texto de Tharp, Estrada, Dalton y Yamauchi (2002). 
aula; sentir una curiosidad por la vida a partir de lo enseñado y que invita a los otros a acercarse a su materia; pero sobre todo, las didactoeugenias se sustentan en el respeto a los estudiantes y al profesor en sí mismo, cuando se generan procesos de mejora en el aprender a partir del enseñar ${ }^{15}$, lo que se relaciona con los cuatros momentos de esta acción didáctica: diagnóstico, planeación, realización y evaluación.

15 Para ampliar las mejores prácticas docentes, se sugieren los textos de Bain (2005) y de Biggs (2006) que pueden adaptarse a otros niveles escolares. 


\section{REFERENCIAS}

ALSINA, A. y PLANAS, N., Matemáticas inclusivas. Propuesta para una educación matemática accesible, Narcea, Madrid, 2008.

BAIN, K., Lo que hacen los mejores profesores universitarios, Universidad de Valencia. España, 2005.

BIGGS, J., Calidad del aprendizaje universitario, Segunda edición, Narcea, España, 2006.

CONTRERAS, J., Enseñanza, currículum y profesorado, Colección Universitaria, Akal, España, 1990.

CASTEJÓN, J.L. y NAVAS, L. (eds.), Aprendizaje, desarrollo y disfunciones. Implicaciones para la enseñanza en la educación secundaria , ECU. España, 2009.

CUKIER, J., «Didactogenia», Contexto Educativo. Revista digital de investigación y nuevas tecnologías, Argentina, 2000.

GADOTI, M., Perspectivas actuales de la educación, Siglo XXI, México, 2003.

GENTO, S. (coord.), Evaluación y estimación del rendimiento académico en el tratamiento educativo de la diversidad, UNED, Madrid, 2010.

PACIOS, S., Introducción a la didáctica, Kapelusz, Madrid, 1980.

RODRÍGUEZ, A., La inteligencia en educación infantil articulada por la herencia, el ambiente escolar, social y familiar, Liber Factory, Madrid, 2014.

ROSARIO MUÑOZ, J.M., Los saberes del profesor para transformar su práctica docente por competencias, Palibrio, EUA, 2011. 
TOMÁS I FOLCH, M., Reconstruir la universidad a través del cambio cultural, UAB, España, 2007.

THARP, R.; ESTRADA, P.; DALTON, S. y YAMAUCHI, L., La actividad en la teoría y en el aula, Paidós, Barcelona, 2002.

ORANTES, A., «Apuntes de Psicología de la Instrucción. Un enfoque analítico», Cuaderno de Posgrado, n. 32, Facultad de Humanidades y Educación, Universidad Central de Venezuela, Venezuela, 2003.

PLA I MOLINS, M., Currículo y educación. Campo semántico de la didáctica, Colección UB 15, Ediciones Universitarias de Barcelona, España, 1997. 